\title{
LIPSCHITZ METRIC FOR CONSERVATIVE SOLUTIONS OF THE TWO-COMPONENT CAMASSA-HOLM SYSTEM
}

\author{
HONG CAI, GENG CHEN, AND YANNAN SHEN
}

\begin{abstract}
In this paper, we construct a Finsler type distance that renders Lipschitz continuous the flow of energy conservative Hölder continuous solutions to the two-component Camassa-Holm system.
\end{abstract}

Keywords. Two-component Camassa-Holm system; Lipschitz metric; conservative solution.

\section{INTRODUCTION}

Consider the Cauchy problem for the two-component Camassa-Holm system

$$
\left\{\begin{array}{l}
u_{t}+\left(\frac{u^{2}}{2}\right)_{x}+P_{x}=0, \\
\rho_{t}+(u \rho)_{x}=0
\end{array}\right.
$$

with initial data

$$
u(0, x)=u_{0}(x) \in H^{1}, \quad \rho(0, x)=\rho_{0}(x) \in L^{2},
$$

where the nonlocal source term $P$ is defined as a convolution

$$
P:=\frac{1}{2} e^{-|x|} *\left(u^{2}+\frac{u_{x}^{2}+\rho^{2}}{2}\right) .
$$

Here $u=u(t, x)$ represents the horizontal velocity of the fluid and $\rho=\rho(t, x)$ is related to the horizontal deviation of the free surface from equilibrium. The system (1.1) was recently derived by Constantin and Ivanov [5] in the context of shallow water theory. The existence of solution was proved by different groups using different methods, c.f. [7, 8, 10, 11. Recently, Grunert, Holden and Raynaud [7] proved existence of a global conservative solution of the Cauchy problem for (1.1) on the line, allowing for nonvanishing and distinct asymptotics at plus and minus infinity. Then in [8] they introduced $\alpha$-dissipative solutions, that provided a continuous interpolation between conservative and dissipative solutions of the Cauchy problem for (1.1) on the line with vanishing asymptotics. On the other hand, the uniqueness of global weak solution was established in [13] by Li and Zhang, who also proved a generic regularity result in [12] using similar method in [1].

However, the solution flows are in general not Lipschitz continuous under the Sobolev metric naturally given by the energy, due to the finite time gradient blowup (energy concentration). A very natural idea is to use a transport metric. In [9], Grunert, Holden and Raynaud established a transport metric under which the solution flows are Lipschitz, where their construction and Lipschitz estimates are via analysis on a semi-linear system on some new coordinates.

In this paper, we establish a new Finsler type transport metric which provides another way in proving the Lipschitz continuous dependence of weak conservative solutions on $H^{1} \times L^{2}$ initial data for the general Cauchy problem (1.1)-(1.3). Since in our construction we directly work on the solution map on the $(x, t)$-coordinates instead of other coordinates, it could reveal some natural ideas used in other kinds of transport metrics from a new point of view. Especially we expect that the new construction could be easily understood by readers in broader fields of research. The method in this paper is very robust. Similar metrics were established for other models whose solutions include cusp singularities, such as the variational wave equation and Novikov equation, [2, 3, 4,

2010 Mathematics Subject Classification. 35Q53; 35B35; 35L60. 
1.1. Previous existence and uniqueness results. For smooth solutions, we differentiate (1.1) with respect to $x$ and use the identity $p_{x x} * f=p * f-f$ for $p=\frac{1}{2} e^{-|x|}, f \in L^{2}(\mathbb{R})$, to get

$$
u_{x t}+u u_{x x}+\frac{1}{2} u_{x}^{2}-u^{2}-\frac{1}{2} \rho^{2}+P=0,
$$

from which one can derive the energy law

$$
\left(\frac{u_{x}^{2}}{2}+\frac{u^{2}}{2}+\frac{\rho^{2}}{2}\right)_{t}+\left(\frac{u u_{x}^{2}}{2}+\frac{u \rho^{2}}{2}+u P\right)_{x}=0 .
$$

Hence, for smooth solutions, the total energy

$$
\mathcal{E}(t):=\int_{\mathbb{R}}\left(u^{2}+u_{x}^{2}+\rho^{2}\right)(t, x) d x=\mathcal{E}(0)
$$

is conserved. And we are able to estimate $P(t)$ and $P_{x}(t)$ by the total energy as follows.

$$
\begin{aligned}
& \|P(t)\|_{L^{\infty}},\left\|P_{x}(t)\right\|_{L^{\infty}} \leq\left\|\frac{1}{2} e^{-|x|}\right\|_{L^{\infty}}\left\|u^{2}+\frac{u_{x}^{2}+\rho^{2}}{2}\right\|_{L^{1}} \leq \frac{1}{2} \mathcal{E}(0), \\
& \|P(t)\|_{L^{2}},\left\|P_{x}(t)\right\|_{L^{2}} \leq\left\|\frac{1}{2} e^{-|x|}\right\|_{L^{2}}\left\|u^{2}+\frac{u_{x}^{2}+\rho^{2}}{2}\right\|_{L^{1}} \leq \frac{1}{\sqrt{2}} \mathcal{E}(0) .
\end{aligned}
$$

Following [7, 13, we introduce the definition of a conservative solution of (1.1)-(1.3).

Definition 1.1. (1) For any $T>0$, by a solution of the Cauchy problem (1.1)-(1.3) on $[0, T]$, we mean a Hölder continuous function $u=u(t, x)$ defined on $[0, T] \times \mathbb{R}$ and $\rho(t, x) \in$ $L^{\infty}\left(0, T ; L^{2}(\mathbb{R})\right)$ with following properties. At each fixed $t$ we have $u(t, \cdot) \in H^{1}(\mathbb{R})$. Moreover, the map $t \mapsto u(t, \cdot)$ is Lipschitz continuous from $[0, T]$ into $L^{2}(\mathbb{R})$ and satisfies the initial condition (1.2) together with $\frac{d}{d t} u=-u u_{x}-P_{x}$ for a.e. $t$. Here equality is understood in the sense of functions in $L^{2}(\mathbb{R})$. The equation $(1.1)_{2}$ holds in the sense of distribution.

(2) A solution $(u, \rho)(t, x)$ is conservative if $\varpi=u_{x}^{2}+\rho^{2}$ provides a distributional solution to the following balance law $\varpi_{t}+(u \varpi)_{x}=2\left(u^{2}-P\right) u_{x}$.

Now, we review the main results on the existence and uniqueness of conservative solutions to the two-component Camassa-Holm system, c.f. [7, 11, 13].

Theorem 1.1. [7, 11, 13] For any initial data $u_{0} \in H^{1}(\mathbb{R}), \rho_{0} \in L^{2}(\mathbb{R})$, the two-component Camassa-Holm system (1.1)-1.3) admits a unique conservative solution $u=u(t, x), \rho=\rho(t, x)$. More precisely, there exists a family of Radon measures $\left\{\mu_{(t)} ; t \in \mathbb{R}_{+}\right\}$, depending continuously on time w.r.t the topology of weak convergence of measures, such that the following properties hold.

(i) The functions $u$ and $\rho$ provide a solution to (1.1) - 1.3 in the sense of Definition 1.1.

(ii) There exists a null set $A \subset \mathbb{R}$ with meas $(A)=0$ such that for every $t \notin A$ the measure $\mu_{(t)}$ is absolutely continuous and has density $u_{x}^{2}(t, \cdot)+\rho^{2}(t, \cdot)$ with respect to Lebesgue measure.

(iii) The family $\left\{\mu_{(t)} ; t \in \mathbb{R}_{+}\right\}$provides a measure-valued solution $\varpi$ to the linear transport equation with source $\varpi_{t}+(u \varpi)_{x}=2\left(u^{2}-P\right) u_{x}$.

1.2. Our main result. Then we come to state our main Lipschitz continuously dependence theorem.

Theorem 1.2. Then let $\left(u_{0}, \rho_{0}\right)$ and $\left(\hat{u}_{0}, \hat{\rho}_{0}\right)$ be two $H^{1}(\mathbb{R}) \times L^{1}(\mathbb{R})$ initial data. Then for every $t \in[0, T]$, for the corresponding solutions $(u, \rho)(t, x)$ and $(\hat{u}, \hat{\rho})(t, x)$, given in Definition 1.1 and Theorem 1.1. we define a geodesic distance $d((u(t), \rho(t)),(\hat{u}(t), \hat{\rho}(t)))$ in Definition 4.2 later, which satisfies

$$
d((u(t), \rho(t)),(\hat{u}(t), \hat{\rho}(t))) \leq C \cdot d\left(\left(u_{0}, \rho_{0}\right),\left(\hat{u}_{0}, \hat{\rho}_{0}\right)\right),
$$

where the constant $C>0$ depends only on $T$ and $E_{1}$.

To prove this theorem, we use three steps. First, in Section 2, we construct a Finsler norm on the tangent vector and show how the norm evolves in time for smooth solutions. In this step, the key estimate (Proposition 2.1) is to prove that the solution is uniform Lipschitz 
continuous under that norm even when approaching singularity. This helps us in the next step to pass through the singularities. In Section 3, we extend the Lipschitz metric to piecewise smooth solutions, where the main result is in Proposition 3.1. Finally, in Section 4, we prove the Theorem 1.2, by extending the Lipschitz metric to general weak solutions using the generic regularity result in [12, which very roughly speaking, shows that the set of piecewise smooth solutions is dense in the set of general weak solutions $(u(t \cdot), \rho(t, \cdot))$ in $H^{1} \times L^{2}$.

\section{The NORM OF TANGENT VECTOR FOR SMOOTH SOLUtion}

In this section, we shall establish a Finsler norm on tangent vector, related to an energy transportation cost. Then by elaborate estimates, we obtain the key result, which, describing how the norm of a tangent vector grows in time for smooth solutions. To this end, let $(u, \rho)(t, x)$ be a smooth solution to (1.1). Consider a family of perturbed solutions of the form

$$
u^{\epsilon}(x)=u(x)+\epsilon v(x)+o(\epsilon), \quad \rho^{\epsilon}(x)=\rho(x)+\epsilon \sigma(x)+o(\epsilon) .
$$

A straightforward calculation yields that the first order perturbations $v$ and $\sigma$ satisfiy

$$
\begin{gathered}
v_{t}+u v_{x}+v u_{x}+\frac{1}{2}\left(\int_{x}^{\infty}-\int_{-\infty}^{x}\right) e^{-|x-y|}\left(2 u v+u_{y} v_{y}+\rho \sigma\right)(y) d y=0, \\
\sigma_{t}+u \sigma_{x}+\sigma u_{x}+v \rho_{x}+\rho v_{x}=0 .
\end{gathered}
$$

Differentiating (2.2) with respect to $x$, one obtains

$$
v_{x t}+u v_{x x}+u_{x} v_{x}+v u_{x x}-2 u v-\rho \sigma+\frac{1}{2} \int_{-\infty}^{\infty} e^{-|x-y|}\left(2 u v+u_{y} v_{y}+\rho \sigma\right)(y) d y=0 .
$$

To continue, we need to add a quantity $w(t, x)$ measuring the horizontal shift:

$$
x^{\epsilon}:=x+\epsilon w(x)+o(\epsilon) .
$$

Here $w(t, x)$ is obtained by propagating along characteristics the shifts $w_{0}(x)$ as the initial data. That is, we require that when $x(t)$ is a characteristic starting from $x_{0}$ then $x^{\epsilon}(t)$ is also a characteristic starting from $x_{0}^{\epsilon}$, so

$$
\frac{d}{d t} x^{\epsilon}(t)=u^{\epsilon}\left(x^{\epsilon}\right) \quad \text { when } \quad \frac{d}{d t} x(t)=u(x) .
$$

By (2.1), 2.5) and taking limit $\epsilon \rightarrow 0$, we have

$$
w_{t}+u w_{x}=v+u_{x} w .
$$

Thus, the Finsler norm on the space of infinitesimal tangent vectors $v$ and $\sigma$ and the $(u, \rho)$ flow itself takes the form

$$
\begin{aligned}
\|(v, \sigma)\|_{(u, \rho)} & :=\inf _{w \in \mathcal{A}}\|(w, v, \sigma)\|_{(u, \rho)} \\
= & \inf _{w \in \mathcal{A}} \int_{\mathbb{R}}\left\{|w|\left(1+u_{x}^{2}+\rho^{2}\right)+\left|v+u_{x} w\right|\left(1+u_{x}^{2}+\rho^{2}\right)+\left|\sigma+\rho_{x} w+\rho w_{x}\right|\right. \\
& \left.\quad \quad \quad\left|2 u_{x}\left(v_{x}+u_{x x} w\right)+2 \rho\left(\sigma+\rho_{x} w\right)+\left(u_{x}^{2}+\rho^{2}\right) w_{x}\right|\right\} e^{-|x|} d x \\
= & \inf _{w \in \mathcal{A}}\left(I_{1}+I_{2}+I_{3}+I_{4}\right) .
\end{aligned}
$$

where $\mathcal{A}=\left\{\right.$ solutions $w(t, x)$ of $(2.6)$ with smooth initial data $\left.w_{0}(x)\right\}$. Here $I_{1} \sim I_{4}$ can be explained as the following.

- $I_{1}$ measures [change in $\left.x\right]\left(1+u_{x}^{2}+\rho^{2}\right)$, where

$$
\text { [change in } x]=\lim _{\epsilon \rightarrow 0} \epsilon^{-1}\left[x^{\epsilon}-x\right]=w(x) .
$$

- $I_{2}$ measures [change in $\left.u\right]\left(1+u_{x}^{2}+\rho^{2}\right)$, where

$$
\text { [change in } u]=\lim _{\epsilon \rightarrow 0} \epsilon^{-1}\left[u^{\epsilon}\left(x^{\epsilon}\right)-u(x)\right]=v(x)+u_{x}(x) w(x) .
$$

- $I_{3}$ measures [change in the base measure with density $\rho$ ], which is the $o(\epsilon)$ term of

$$
\rho^{\epsilon}\left(x^{\epsilon}\right) d x^{\epsilon}-\rho(x) d x .
$$


- $I_{4}$ measures [change in the base measure with density $u_{x}^{2}+\rho^{2}$ ], which is the $o(\epsilon)$ term of

$$
\left(\left(u_{x}^{\epsilon}\left(x^{\epsilon}\right)\right)^{2}+\left(\rho^{\epsilon}\left(x^{\epsilon}\right)\right)^{2}\right) d x^{\epsilon}-\left(u_{x}^{2}(x)+\rho^{2}(x)\right) d x .
$$

Proposition 2.1. Let $(u, \rho)$ be a smooth solution to (1.1)-(1.3), and assume that the first order perturbations $(v, \sigma)$ satisfy the equations (2.2)-2.3). Then for any $\tau>0$, we have

$$
\|(v, \sigma)(\tau)\|_{(u, \rho)(\tau)} \leq e^{C \tau}\|(v, \sigma)(0)\|_{\left(u_{0}, \rho_{0}\right)},
$$

for some constant $C>0$ depending only on initial total energy.

Proof. It suffices to show that

$$
\frac{d}{d t}\|(w, v, \sigma)(t)\|_{(u, \rho)(t)} \leq C\|(w, v, \sigma)(t)\|_{(u, \rho)(t)},
$$

for any $w, v$ and $\sigma$ satisfying (2.6), (2.2) and (2.3), respectively. Here and in this section, $C$ is a constant depending only on initial total energy which may vary in different estimates. First,

$$
\frac{d}{d t} \int_{\mathbb{R}}|f| e^{-|x|} d x=\int_{\mathbb{R}}\left(|f| e^{-|x|}\right)_{t}+\left(u|f| e^{-|x|}\right)_{x} d x \leq \int_{\mathbb{R}}\left|f_{t}+(u f)_{x}\right| e^{-|x|} d x+\|u\|_{L^{\infty}} \int_{\mathbb{R}}|f| e^{-|x|} d x
$$

for any smooth function $f$. Hence, we only have to focus on how to estimate $f_{t}+(u f)_{x}$ with $f$ being the integrands without $e^{-|x|}$ in $I_{1} \sim I_{4}$. For convenience, we drop all the $e^{-|x|}$ terms in our calculation in both $\left|f_{t}+(u f)_{x}\right| e^{-|x|}$ and $I_{1} \sim I_{4}$. This does not impact our result.

(1). To estimate the time derivative of $I_{1}$, from $(1.1)_{2}$ and $(1.4)$, it follows

$$
\left(1+u_{x}^{2}+\rho^{2}\right)_{t}+\left(u\left(1+u_{x}^{2}+\rho^{2}\right)\right)_{x}=u_{x}+2 u^{2} u_{x}-2 u_{x} P .
$$

Then by (2.6) and (2.10), we have

$$
\begin{aligned}
& {\left[w\left(1+u_{x}^{2}+\rho^{2}\right)\right]_{t}+\left[u w\left(1+u_{x}^{2}+\rho^{2}\right)\right]_{x} } \\
= & \left(w_{t}+u w_{x}\right)\left(1+u_{x}^{2}+\rho^{2}\right)+w\left[\left(1+u_{x}^{2}+\rho^{2}\right)_{t}+\left(u\left(1+u_{x}^{2}+\rho^{2}\right)\right)_{x}\right] \\
= & \left(v+u_{x} w\right)\left(1+u_{x}^{2}+\rho^{2}\right)+w\left(u_{x}+2 u^{2} u_{x}-2 u_{x} P\right) .
\end{aligned}
$$

This yields the estimate

$$
\frac{d}{d t} \int_{\mathbb{R}}|w|\left(1+u_{x}^{2}+\rho^{2}\right) d x \leq \int_{\mathbb{R}}\left|v+u_{x} w\right|\left(1+u_{x}^{2}+\rho^{2}\right) d x+C \int_{\mathbb{R}}|w|\left(1+u_{x}^{2}+\rho^{2}\right) d x .
$$

(2). To estimate the time derivative of $I_{2}$, recalling (1.4), 2.2), 2.6) and 2.10), we obtain

$$
\begin{aligned}
& {\left[\left(v+u_{x} w\right)\left(1+u_{x}^{2}+\rho^{2}\right)\right]_{t}+\left[u\left(v+u_{x} w\right)\left(1+u_{x}^{2}+\rho^{2}\right)\right]_{x} } \\
= & {\left[v_{t}+u v_{x}+u_{x}\left(w_{t}+u w_{x}\right)+w\left(u_{x t}+u u_{x x}\right)\right]\left(1+u_{x}^{2}+\rho^{2}\right) } \\
& +\left(v+u_{x} w\right)\left[\left(1+u_{x}^{2}+\rho^{2}\right)_{t}+\left(u\left(1+u_{x}^{2}+\rho^{2}\right)\right)_{x}\right] \\
= & {\left[\frac{1}{2}\left(u_{x}^{2} w+\rho^{2} w\right)-\frac{1}{2}\left(\int_{x}^{\infty}-\int_{-\infty}^{x}\right) e^{-|x-y|}\left(2 u v+u_{y} v_{y}+\rho \sigma\right)(y) d y\right]\left(1+u_{x}^{2}+\rho^{2}\right) } \\
& +w\left(u^{2}-P\right)\left(1+u_{x}^{2}+\rho^{2}\right)+\left(v+u_{x} w\right)\left(u_{x}+2 u^{2} u_{x}-2 u_{x} P\right) .
\end{aligned}
$$

The first term on the right hand side of $(2.12)$ may be rewritten as

$$
\begin{aligned}
\frac{1}{2}\left(u_{x}^{2} w+\rho^{2} w\right)(x)= & -\frac{1}{2}\left(\int_{x}^{\infty}-\int_{-\infty}^{x}\right)\left(e^{-|x-y|} \frac{u_{y}^{2} w+\rho^{2} w}{2}(y)\right)_{y} d y \\
= & -\frac{1}{2}\left(\int_{x}^{\infty}-\int_{-\infty}^{x}\right) e^{-|x-y|}\left(u_{y} u_{y y} w+\rho \rho_{y} w+\frac{u_{y}^{2}+\rho^{2}}{2} w_{y}\right)(y) d y \\
& +\frac{1}{2} \int_{-\infty}^{\infty} e^{-|x-y|} \frac{u_{y}^{2} w+\rho^{2} w}{2}(y) d y
\end{aligned}
$$

Note that

$$
2 u v=2 u\left(v+u_{y} w\right)-2 u u_{y} w
$$


which, in combination with 2.13 and the Sobolev inequality that

$$
\begin{aligned}
& \quad\left|\int_{\mathbb{R}}\left[\frac{1}{2}\left(u_{x}^{2} w+\rho^{2} w\right)-\frac{1}{2}\left(\int_{x}^{\infty}-\int_{-\infty}^{x}\right) e^{-|x-y|}\left(2 u v+u_{y} v_{y}+\rho \sigma\right)(y) d y\right]\left(1+u_{x}^{2}+\rho^{2}\right)(x) d x\right| \\
& \leq C \int_{\mathbb{R}} \int_{\mathbb{R}} e^{-|x-y|}\left(1+u_{x}^{2}+\rho^{2}\right)(x) d x\left[\left|2 u\left(v+u_{y} w\right)-2 u u_{y} w\right|+\left|u_{y}^{2} w+\rho^{2} w\right|\right. \\
& \left.\quad+\left|2 u_{y}\left(v_{y}+u_{y y} w\right)+2 \rho\left(\sigma+\rho_{y} w\right)+\left(u_{y}^{2}+\rho^{2}\right) w_{y}\right|\right](y) d y \\
& \leq C \int_{\mathbb{R}}\left|v+u_{x} w\right|\left(1+u_{x}^{2}+\rho^{2}\right) d x+C \int_{\mathbb{R}}|w|\left(1+u_{x}^{2}+\rho^{2}\right) d x \\
& \quad+C \int_{\mathbb{R}}\left|2 u_{x}\left(v_{x}+u_{x x} w\right)+2 \rho\left(\sigma+\rho_{x} w\right)+\left(u_{x}^{2}+\rho^{2}\right) w_{x}\right| d x .
\end{aligned}
$$

Consequently, in accordance with 2.12 and 2.14 , we derive

$$
\frac{d I_{2}}{d t}=\frac{d}{d t} \int_{\mathbb{R}}\left|v+u_{x} w\right|\left(1+u_{x}^{2}+\rho^{2}\right) d x \leq C\left(I_{1}+I_{2}+I_{4}\right) .
$$

(3). For the time derivative of $I_{3}$, we first differentiate (2.6) with respect to $x$,

$$
w_{x t}+\left(u w_{x}\right)_{x}=v_{x}+u_{x x} w+u_{x} w_{x} .
$$

With this help, utilizing the estimates $(1.1)_{2}$ and $(2.3)$, we can derive

$$
\begin{aligned}
& {\left[\sigma+\rho_{x} w+\rho w_{x}\right]_{t}+\left[u\left(\sigma+\rho_{x} w+\rho w_{x}\right)\right]_{x} } \\
= & \sigma_{t}+(u \sigma)_{x}+\rho_{x}\left(w_{t}+u w_{x}\right)+w\left(\rho_{x t}+\left(u \rho_{x}\right)_{x}\right)+\rho\left(w_{x t}+\left(u w_{x}\right)_{x}\right)+w_{x}\left(\rho_{t}+u \rho_{x}\right) \\
= & -v \rho_{x}-v_{x} \rho+\rho_{x}\left(v+u_{x} w\right)-w\left(u_{x} \rho_{x}+u_{x x} \rho\right)+\rho\left(v_{x}+u_{x x} w+u_{x} w_{x}\right)-w_{x} u_{x} \rho \\
= & 0
\end{aligned}
$$

which implies

$$
\frac{d I_{3}}{d t}=\frac{d}{d t} \int_{\mathbb{R}}\left|\sigma+\rho_{x} w+\rho w_{x}\right| d x \leq 0 .
$$

(4). We repeat the same procedure on $I_{4}$. Differentiating (1.4) with respect to $x$ to get

$$
u_{x x t}+\left(u u_{x x}\right)_{x}+u_{x} u_{x x}-2 u u_{x}-\rho \rho_{x}+P_{x}=0 .
$$

Using (1.1) 2 , (1.4), (2.3), (2.4), 2.6), 2.16) and (2.18), one has

$$
\begin{aligned}
& {\left[2 u_{x}\left(v_{x}+u_{x x} w\right)+2 \rho\left(\sigma+\rho_{x} w\right)+\left(u_{x}^{2}+\rho^{2}\right) w_{x}\right]_{t} } \\
& +\left[u\left(2 u_{x}\left(v_{x}+u_{x x} w\right)+2 \rho\left(\sigma+\rho_{x} w\right)+\left(u_{x}^{2}+\rho^{2}\right) w_{x}\right)\right]_{x} \\
= & 2\left(u_{x t}+u u_{x x}\right)\left(v_{x}+u_{x x} w\right)+2 u_{x}\left[v_{x t}+\left(u v_{x}\right)_{x}+u_{x x}\left(w_{t}+u w_{x}\right)+w\left(u_{x x t}+\left(u u_{x x}\right)_{x}\right]\right. \\
& +2\left(\rho_{t}+u \rho_{x}\right)\left(\sigma+\rho_{x} w\right)+2 \rho\left[\sigma_{t}+(u \sigma)_{x}+\rho_{x}\left(w_{t}+u w_{x}\right)+w\left(\rho_{x t}+\left(u \rho_{x}\right)_{x}\right]\right. \\
& +2 u_{x} w_{x}\left(u_{x t}+u u_{x x}\right)+2 \rho w_{x}\left(\rho_{t}+u \rho_{x}\right)+\left(u_{x}^{2}+\rho^{2}\right)\left(w_{x t}+\left(u w_{x}\right)_{x}\right) \\
= & 2\left(u^{2}-P\right)\left(v_{x}+u_{x x} w+u_{x} w_{x}\right)+4 u u_{x}\left(v+u_{x} w\right)-2 u_{x} w P_{x} \\
& -u_{x} \int_{\mathbb{R}} e^{-|x-y|}\left(2 u v+u_{y} v_{y}+\rho \sigma\right)(y) d y .
\end{aligned}
$$

By virtue of the integration by parts, the first term will be estimated as

$$
\begin{aligned}
& 2 \int_{\mathbb{R}}\left(u^{2}-P\right)\left(v_{x}+u_{x x} w+u_{x} w_{x}\right) d x=2 \int_{\mathbb{R}}\left(u^{2}-P\right)\left(v+u_{x} w\right)_{x} d x \\
= & -2 \int_{\mathbb{R}}\left(u^{2}-P\right)_{x}\left(v+u_{x} w\right) d x \leq C \int_{\mathbb{R}}\left|v+u_{x} w\right|\left(1+u_{x}^{2}+\rho^{2}\right) .
\end{aligned}
$$


Observe that the rightmost term on the right hand side of 2.19 ) can be expressed as

$$
\begin{aligned}
2 u v+u_{y} v_{y}+\rho \sigma= & 2 u\left(v+u_{y} w\right)-2 u u_{y} w+\frac{1}{2}\left[2 u_{y}\left(v_{y}+u_{y y} w\right)\right. \\
& \left.+2 \rho\left(\sigma+\rho_{y} w\right)+\left(u_{y}^{2}+\rho^{2}\right) w_{y}\right]-\frac{1}{2}\left[\left(u_{y}^{2}+\rho^{2}\right) w\right]_{y} .
\end{aligned}
$$

Thus, it holds that,

$$
\begin{aligned}
& \quad\left|-\int_{\mathbb{R}} \int_{\mathbb{R}} e^{-|x-y|}\left(2 u v+u_{y} v_{y}+\rho \sigma\right)(y) d y u_{x}(x) d x\right| \\
& \leq C \int_{\mathbb{R}} \int_{\mathbb{R}}\left|\frac{\partial e^{-|x-y|}}{\partial y} u_{x}\right| d x\left|\left(u_{y}^{2}+\rho^{2}\right) w\right|(y) d y+C \int_{\mathbb{R}} \int_{\mathbb{R}} e^{-|x-y|} u_{x} d x\left[\left|2 u\left(v+u_{y} w\right)-2 u u_{y} w\right|\right. \\
& \left.\quad+\left|2 u_{y}\left(v_{y}+u_{y y} w\right)+2 \rho\left(\sigma+\rho_{y} w\right)+\left(u_{y}^{2}+\rho^{2}\right) w_{y}\right|\right](y) d y \\
& \leq C \int_{\mathbb{R}}|w|\left(1+u_{x}^{2}+\rho^{2}\right) d x+C \int_{\mathbb{R}}\left|v+u_{x} w\right|\left(1+u_{x}^{2}+\rho^{2}\right) d x \\
& \quad+C \int_{\mathbb{R}}\left|2 u_{x}\left(v_{x}+u_{x x} w\right)+2 \rho\left(\sigma+\rho_{x} w\right)+\left(u_{x}^{2}+\rho^{2}\right) w_{x}\right| d x,
\end{aligned}
$$

where we have used the Hölder and Sobolev inequalities. Thanks to 2.19 -2.21 , we obtain

$$
\frac{d I_{4}}{d t}=\frac{d}{d t} \int_{\mathbb{R}}\left|2 u_{x}\left(v_{x}+u_{x x} w\right)+2 \rho\left(\sigma+\rho_{x} w\right)+\left(u_{x}^{2}+\rho^{2}\right) w_{x}\right| d x \leq C\left(I_{1}+I_{2}+I_{4}\right) .
$$

Thus, putting the estimates 2.11), 2.15), 2.17) and (2.22) together, we obtain the desired inequality (2.9). In turn, this will yield (2.8). This completes the proof of Theorem 2.1.

\section{METRIC FOR PIECEWISE SMOOTH SOLUTIONS}

3.1. Length of a path of solutions in transformed coordinates. For any path $\theta \mapsto$ $\left(u^{\theta}, \rho^{\theta}\right), \theta \in[0,1]$ of smooth solutions to 1.1 , the analysis in the previous section has provided an estimate on how its weighted length increases in time. However, even for smooth initial data, the quantity $u_{x}$ or $\rho_{x}$ may blow up in finite time, c.f. [6, 10]. When singularity forms, the norm $\|(v, \sigma)\|_{(u, \rho)}$ may no longer exist, even if it does exist, it is not obvious that the estimate 2.8 holds. Therefore, we examine these issues in this subsection. Following the idea in [12], we introduce new coordinates $(t, \xi)$, where $\xi$ is implicitly defined as

$$
\int_{0}^{\bar{y}(\xi)}\left(1+u_{0, x}^{2}+\rho_{0}^{2}\right) d x=\xi
$$

The characteristic is corresponding to the curve on which $\xi$ equals to a constant,

$$
\partial_{t} y(t, \xi)=u(t, y(t, \xi)), \quad y(0, \xi)=\bar{y}(\xi)
$$

In terms of these variables, we denote

$$
q=\left(1+u_{x}^{2}+\rho^{2}\right) \cdot \frac{\partial y}{\partial \xi}, \quad L=\frac{q}{1+u_{x}^{2}+\rho^{2}}, \quad \alpha=\frac{u_{x} q}{1+u_{x}^{2}+\rho^{2}}, \quad \beta=\frac{\rho q}{1+u_{x}^{2}+\rho^{2}} .
$$

Then one obtains a semi-linear system

$$
\left\{\begin{array}{l}
u_{t}=-P_{x} \\
q_{t}=\alpha\left(1+2 u^{2}-2 P\right) \\
L_{t}=\alpha \\
\alpha_{t}=-P L+L u^{2}+\frac{1}{2}(q-L) \\
\beta_{t}=0
\end{array}\right.
$$

By expressing the solution $(u, \rho)(t, \xi)$ in terms of the original variables $(t, x)$, one obtains a solution of the Cauchy problem [1.1)-11.3). Indeed, we have the following results, c.f. [7, 13]. 
Lemma 3.1. Let $(x, u, L, \alpha, \beta, q)(t, \xi)$ be a smooth solution to the system (3.1) -(3.4) with $q>0$. Then the functions $(u, \rho)(t, x)$ whose graph is

$$
\operatorname{Graph}(u, \rho):=\left\{(t, x(t, \xi), u(t, \xi), \rho(t, \xi)) ; \quad(t, \xi) \in \mathbb{R}_{+} \times \mathbb{R}\right\}
$$

provide a unique conservative solution to the system (1.1) 1.3 .

For smooth data $\left(u_{0}, \rho_{0}\right)$, the solution $(t, \xi) \mapsto(x, u, L, \alpha, \beta, q)(t, \xi)$ of the semilinear system (3.4) remains smooth on the entire $t-\xi$ plane. Yet, the solution of (1.1) can have singularities because the coordinate change: $(t, \xi) \rightarrow(t, x)$ is not smoothly invertible. Indeed, by definition, its Jacobian matrix

$$
\left(\begin{array}{cc}
x_{\xi} & x_{t} \\
t_{\xi} & t_{t}
\end{array}\right)=\left(\begin{array}{cc}
L & u \\
0 & 1
\end{array}\right)
$$

So, at a point $\left(t_{0}, \xi_{0}\right)$ where $L\left(t_{0}, \xi_{0}\right) \neq 0$, this matrix is invertible, having a strictly positive determinant. The solution of (3.4) is thus smooth on a neighborhood of the point $\left(t_{0}, \xi_{0}\right)=$ $\left(t_{0}, x\left(t_{0}, \xi_{0}\right)\right)$. To study the set of points in $t-x$ plane where the solution is singular, we thus introduce the suitable regularity conditions, c.f. [1, 12].

Definition 3.1. We say that a solution $(u, \rho)(t, x)$ of 1.1 has generic singularities for $t \in$ $[0, T]$ if it admits a representation of the form (3.5), where

(i) the functions $(x, u, L, \alpha, \beta, q)(t, \xi)$ are $\mathcal{C}^{\infty}$,

(ii) for $t \in[0, T]$, the following generic conditions hold

$$
\begin{array}{ll}
\left(\mathrm{G}_{1}\right) . & L=0, L_{\xi}=0, \alpha_{\xi}=0 \Longrightarrow \beta_{\xi} \neq 0, L_{\xi \xi} \neq 0, \alpha_{\xi \xi} \neq 0, \\
\left(\mathrm{G}_{2}\right) . & L=0, L_{\xi}=0, \beta_{\xi}=0 \Longrightarrow \alpha_{\xi} \neq 0, L_{\xi \xi} \neq 0, \beta_{\xi \xi} \neq 0 .
\end{array}
$$

Definition 3.2. We say that a path of initial data $\gamma_{0}: \theta \mapsto\left(u_{0}^{\theta}, \rho_{0}^{\theta}\right), \theta \in[0,1]$ is a piecewise regular path if the following conditions hold

(i) There exists a continuous map $(\xi, \theta) \mapsto(x, u, L, \alpha, \beta, q)$ such that the semilinear system (3.1) - (3.4) holds for $\theta \in[0,1]$, and the function $\left(u^{\theta}, \rho^{\theta}\right)(x, t)$ whose graph is

$$
\operatorname{Graph}\left(u^{\theta}, \rho^{\theta}\right)=\left\{(t, x(t, \xi, \theta), u(t, \xi, \theta), \rho(t, \xi, \theta)) ; \quad(t, \xi) \in \mathbb{R}_{+} \times \mathbb{R}\right\}
$$

provides the conservation solution of (1.1) with initial data $u^{\theta}(0, x)=u_{0}^{\theta}(x), \rho^{\theta}(0, x)=\rho_{0}^{\theta}(x)$.

(ii) There exist finitely many values $0=\theta_{0}<\theta_{1}<\cdots<\theta_{N}=1$ such that the map $(\xi, \theta) \mapsto(x, u, L, \alpha, \beta, q)$ is $\mathcal{C}^{\infty}$ for $\theta \in\left(\theta_{i-1}, \theta_{i}\right), i=1, \cdots, N$, and the solution $\left(u^{\theta}, \rho^{\theta}\right)(t, x)$ has only generic singularities at time $t=0$.

In addition, if for $\theta \in[0,1] \backslash\left\{\theta_{1}, \cdots, \theta_{N}\right\}$, the solution $\left(u^{\theta}, \rho^{\theta}\right)(t)$ has only generic singularities when $t \in[0, T]$, then we say that the path of solution $\gamma_{t}: \theta \mapsto\left(u^{\theta}, \rho^{\theta}\right)$ is piecewise regular for $t \in[0, T]$.

The following result shows that the set of piecewise regular paths is dense, c.f. [12].

Lemma 3.2. [12] Given $T>0$, let $\theta \mapsto\left(x^{\theta}, u^{\theta}, L^{\theta}, \alpha^{\theta}, \beta^{\theta}, q^{\theta}\right), \theta \in[0,1]$, be a smooth path of solutions to the system (3.1)-(3.4). Then there exists a sequence of paths of solution $\theta \mapsto$ $\left(x_{n}^{\theta}, u_{n}^{\theta}, L_{n}^{\theta}, \alpha_{n}^{\theta}, \beta_{n}^{\theta}, q_{n}^{\theta}\right)$, such that

(i) For each $n \geq 1$, the path of corresponding solution of (1.1) $\theta \mapsto\left(u_{n}^{\theta}, \rho_{n}^{\theta}\right)$ is regular for $t \in[0, T]$, according to Definition 3.2.

(ii) For any bounded domain $\Omega$ in the $t-\xi$ space, the functions $\left(x_{n}^{\theta}, u_{n}^{\theta}, L_{n}^{\theta}, \alpha_{n}^{\theta}, \beta_{n}^{\theta}, q_{n}^{\theta}\right)$ converge to $\left(x^{\theta}, u^{\theta}, L^{\theta}, \alpha^{\theta}, \beta^{\theta}, q^{\theta}\right)$ uniformly in $\mathcal{C}^{k}([0,1] \times \Omega)$, for every $k \geq 1$, as $n \rightarrow \infty$.

Our aim now is to show that the weighted length of a regular path satisfies the same estimates as (2.8) for smooth paths. To this end, for a reference solution $(u, \rho)$ of (1.1) and a family of perturbed solutions $\left(u^{\varepsilon}, \rho^{\varepsilon}\right)$, we assume that, in the $t-\xi$ coordinates, these define a family of smooth solutions of (3.1)-(3.4), say $\left(x^{\varepsilon}, u^{\varepsilon}, L^{\varepsilon}, \alpha^{\varepsilon}, \beta^{\varepsilon}, q^{\varepsilon}\right)$. Consider the perturbed solutions

$$
\left(x^{\varepsilon}, u^{\varepsilon}, L^{\varepsilon}, \alpha^{\varepsilon}, \beta^{\varepsilon}, q^{\varepsilon}\right)(t, \xi)=(x, u, L, \alpha, \beta, q)(t, \xi)+\varepsilon(X, U, \mathcal{L}, \mathcal{A}, \mathcal{B}, Q)(t, \xi)+o(\varepsilon) .
$$

By the smooth coefficients of (3.2)-(3.4), we have that the first order perturbations satisfy a linearized system and are well defined for $(t, \xi) \in \mathbb{R}_{+} \times \mathbb{R}$. Similar to the argument of [3, 4], we 
express the quantities $w, v, \sigma$ appearing in 2.7) in terms of $(X, U, \mathcal{L}, \mathcal{A}, \mathcal{B}, Q)$. More specifically, we see that

(1) The shift in $x$ is computed by

$$
w=\lim _{\varepsilon \rightarrow 0} \frac{x^{\varepsilon}\left(t, \xi^{\varepsilon}\right)-x(t, \xi)}{\varepsilon}=X+\left.x_{\xi} \cdot \frac{\partial \xi^{\varepsilon}}{\partial \varepsilon}\right|_{\varepsilon=0} .
$$

(2) We will calculate the change in $u$ as

$$
v+u_{x} w=\lim _{\varepsilon \rightarrow 0} \frac{u^{\varepsilon}\left(t, \xi^{\varepsilon}\right)-u(t, \xi)}{\varepsilon}=U+\left.u_{\xi} \cdot \frac{\partial \xi^{\varepsilon}}{\partial \varepsilon}\right|_{\varepsilon=0} .
$$

(3) To achive the change in base measure with density $\rho$, first, we have

$$
\left.\frac{d}{d \varepsilon} \beta^{\varepsilon}\right|_{\varepsilon=0}=\lim _{\varepsilon \rightarrow 0} \frac{\beta^{\varepsilon}\left(t, \xi^{\varepsilon}\right)-\beta(t, \xi)}{\varepsilon}=\mathcal{B}+\left.\beta_{\xi} \cdot \frac{\partial \xi^{\varepsilon}}{\partial \varepsilon}\right|_{\varepsilon=0} .
$$

Then the integrand in $I_{3}$ is calculated as

$$
\left.\frac{d}{d \varepsilon}\left(\beta^{\varepsilon}+\beta L \cdot \xi_{x}^{\varepsilon}\right)\right|_{\varepsilon=0}=\mathcal{B}+\left.\beta_{\xi} \cdot \frac{\partial \xi^{\varepsilon}}{\partial \varepsilon}\right|_{\varepsilon=0}+\left.\beta L \cdot \frac{\partial \xi_{x}^{\varepsilon}}{\partial \varepsilon}\right|_{\varepsilon=0},
$$

(4) To complete the analysis, we have to concern the term due to the change in base measure with density $u_{x}^{2}+\rho^{2}$. Indeed, it follows

$$
\left.\frac{d}{d \varepsilon}\left(q^{\varepsilon}-L^{\varepsilon}+q L \cdot \xi_{x}^{\varepsilon}-L^{2} \cdot \xi_{x}^{\varepsilon}\right)\right|_{\varepsilon=0}=Q-\mathcal{L}+\left.\left(q_{\xi}-L_{\xi}\right) \cdot \frac{\partial \xi^{\varepsilon}}{\partial \varepsilon}\right|_{\varepsilon=0}+\left.(q-L) L \cdot \frac{\partial \xi_{x}^{\varepsilon}}{\partial \varepsilon}\right|_{\varepsilon=0},
$$

where

and

$$
\left.\frac{d}{d \varepsilon} q^{\varepsilon}\right|_{\varepsilon=0}=\lim _{\varepsilon \rightarrow 0} \frac{q^{\varepsilon}\left(t, \xi^{\varepsilon}\right)-q(t, \xi)}{\varepsilon}=Q+\left.q_{\xi} \cdot \frac{\partial \xi^{\varepsilon}}{\partial \varepsilon}\right|_{\varepsilon=0},
$$

Notice that

$$
\left.\frac{d}{d \varepsilon} L^{\varepsilon}\right|_{\varepsilon=0}=\lim _{\varepsilon \rightarrow 0} \frac{L^{\varepsilon}\left(t, \xi^{\varepsilon}\right)-L(t, \xi)}{\varepsilon}=\mathcal{L}+\left.L_{\xi} \cdot \frac{\partial \xi^{\varepsilon}}{\partial \varepsilon}\right|_{\varepsilon=0}
$$

$$
\left(1+u_{x}^{2}+\rho^{2}\right) d x=q d \xi
$$

Thus, from the above analysis, we see that the weighted norm of a tangent vector (2.7) can be written as

$$
\|(w, v, \sigma)\|_{(u, \rho)}=\sum_{\ell=1}^{4} \int_{\mathbb{R}}\left|J_{\ell}(t, \xi)\right| d \xi,
$$

where

$$
\begin{aligned}
& J_{1}=\left(X+\left.x_{\xi} \cdot \frac{\partial \xi^{\varepsilon}}{\partial \varepsilon}\right|_{\varepsilon=0}\right) q e^{-|y(t, \xi)|}, \quad J_{2}=\left(U+\left.u_{\xi} \cdot \frac{\partial \xi^{\varepsilon}}{\partial \varepsilon}\right|_{\varepsilon=0}\right) q e^{-|y(t, \xi)|}, \\
& J_{3}=\left(\mathcal{B}+\left.\beta_{\xi} \cdot \frac{\partial \xi^{\varepsilon}}{\partial \varepsilon}\right|_{\varepsilon=0}+\left.\beta L \cdot \frac{\partial \xi_{x}^{\varepsilon}}{\partial \varepsilon}\right|_{\varepsilon=0}\right) e^{-|y(t, \xi)|}, \\
& J_{4}=\left(Q-\mathcal{L}+\left.\left(q_{\xi}-L_{\xi}\right) \cdot \frac{\partial \xi^{\varepsilon}}{\partial \varepsilon}\right|_{\varepsilon=0}+\left.(q-L) L \cdot \frac{\partial \xi_{x}^{\varepsilon}}{\partial \varepsilon}\right|_{\varepsilon=0}\right) e^{-|y(t, \xi)|} .
\end{aligned}
$$

Since $\xi$ or $\xi^{\varepsilon}$ equals to a constant along the characteristic, and the horizontal shift, given in 2.5 - 2.6), transports one characteristic to another characteristic, we have $\frac{\partial \xi^{\varepsilon}}{\partial \varepsilon}(t)=\frac{\partial \xi^{\varepsilon}}{\partial \varepsilon}(0)$. Then, it is clear that each integrand $J_{\ell}$ is continuous, for $\ell=1,2,3,4$.

Now, we are ready to define the length of the piecewise regular path.

Definition 3.3. The length $\left\|\gamma_{t}\right\|$ of the piecewise regular path $\gamma_{t}: \theta \mapsto\left(u^{\theta}, \rho^{\theta}\right)(t)$ is defined as

$$
\left\|\gamma_{t}\right\|=\inf _{\gamma_{t}} \int_{0}^{1} \sum_{\ell=1}^{4} \int_{\mathbb{R}}\left|J_{\ell}^{\theta}(t, \xi)\right| d \xi d \theta,
$$

where the infimum is taken over all piecewise regular path.

Then one could prove that the appearance of the generic singularity will not impact the Lipschitz property of the metric as given following, which extends the Lipschitz property in Proposition 2.1 to piecewise smooth solutions with generic singularities. 
Proposition 3.1. Given any $T>0$, consider a path of solutions $\theta \mapsto\left(u^{\theta}, \rho^{\theta}\right)$ of (1.1), which is piecewise regular for $t \in[0, T]$. Moreover, the total energy is less than a constant $E_{1}>0$. Then there exists some constant $C>0$, such that

$$
\left\|\gamma_{t}\right\| \leq C\left\|\gamma_{0}\right\|
$$

where the constant $C$ depends only on $T$ and $E_{1}$.

The proof is similar to [4, we omit it here for brevity.

\section{Construction of the geodesic distance for general Weak solution}

Our ultimate goal is to show that the flow generated by the two-component Camassa-Holm (1.1) - 1.3 is Lipschitz continuous with respect to the geodesic distance defined in Definition 4.2 First, in light of [12], for an open dense set of initial data $\mathcal{D} \subset\left\{\left(u_{0}, \rho_{0}\right) ; u_{0} \in \mathcal{C}^{3}(\mathbb{R}) \cap\right.$ $\left.H^{1}(\mathbb{R}), \rho_{0} \in \mathcal{C}^{2}(\mathbb{R}) \cap L^{2}(\mathbb{R})\right\}$, the corresponding solution $(u, \rho)(t, x)$ of $(1.1)$ is piecewise smooth, with singularities occurring in finitely many isolated points. Now, on $\mathcal{D}^{\infty}:=\left(\mathcal{C}_{0}^{\infty} \times \mathcal{C}_{0}^{\infty}\right) \cap \mathcal{D}$, we construct a geodesic distance.

Let two data $(u, \rho),(\hat{u}, \hat{\rho}) \in \mathcal{D}^{\infty}$ be given. Then fix any constant $E_{1}>0$, denote the set

$$
\Sigma:=\left\{u \in H^{1}(\mathbb{R}), \rho \in L^{2}(\mathbb{R}) ; \mathcal{E}(u, \rho):=\int_{\mathbb{R}}\left(u^{2}+u_{x}^{2}+\rho^{2}\right)(x) d x \leq E_{1}\right\} .
$$

Definition 4.1. For solutions with initial data in $\mathcal{D}^{\infty} \cap \Sigma$, we define the geodesic distance $d((u, \rho),(\hat{u}, \hat{\rho}))$ as the infimum among the weighted lengths of all piecewise regular paths $\theta \mapsto$ $\left(u^{\theta}, \rho^{\theta}\right)$, which connect $(u, \rho)$ with $(\hat{u}, \hat{\rho})$, that is, for any time $t$,

$$
\begin{array}{r}
d((u, \rho),(\hat{u}, \hat{\rho})):=\inf \left\{\left\|\gamma_{t}\right\| ; \gamma_{t} \text { is a piecewise regular path, } \gamma_{t}(0)=(u, \rho),\right. \\
\left.\gamma_{t}(1)=(\hat{u}, \hat{\rho}), \mathcal{E}\left(u^{\theta}, \rho^{\theta}\right) \leq E_{1} \text { for all } \theta \in[0,1]\right\} .
\end{array}
$$

Now, we can define the metric for the general weak solutions, using the result in Lemma 3.2 .

Definition 4.2. Let $\left(u_{0}, \rho_{0}\right)$ and $\left(\hat{u}_{0}, \hat{\rho}_{0}\right)$ in $H^{1}(\mathbb{R}) \times L^{1}(\mathbb{R})$ be two initial data as required in the existence Theorem 1.1. Denote $(u, \rho)$ and $(\hat{u}, \hat{\rho})$ to be the corresponding global weak solutions, then we define, for any time $t$,

$$
d((u, \rho),(\hat{u}, \hat{\rho})):=\lim _{n \rightarrow \infty} d\left(\left(u^{n}, \rho^{n}\right),\left(\hat{u}^{n}, \hat{\rho}^{n}\right)\right),
$$

for any two sequences of solutions $\left(u^{n}, \rho^{n}\right)$ and $\left(\hat{u}^{n}, \hat{\rho}^{n}\right)$ in $\mathcal{D}^{\infty} \cap \Sigma$ with

$$
\left\|u^{n}-u\right\|_{H^{1}} \rightarrow 0,\left\|\rho^{n}-\rho\right\|_{L^{2}} \rightarrow 0 \quad \text { and } \quad\left\|\hat{u}^{n}-\hat{u}\right\|_{H^{1}} \rightarrow 0, \mid \hat{\rho}^{n}-\hat{\rho} \|_{L^{2}} \rightarrow 0 .
$$

The limit in the definition is independent on the selection of sequences, because the solution flows are Lipschitz in $\mathcal{D}^{\infty} \cap \Sigma$, so the definition is well-defined. Since the concatenation of two piecewise regular paths is still a piecewise regular path (after a suitable re-parameterization), it is clear that $d(\cdot, \cdot)$ is a distance. By the fact that $\mathcal{D}^{\infty} \cap \Sigma$ is a dense set in the solution space, which is given in Lemma 3.2, one could easily extend the Lipschitz metric to the general initial data. Thus, by virtue of Proposition 3.1, we report directly the proof of Theorem 1.2 .

Similar as in [4], we have

Proposition 4.1. For any $(u, \rho),(\hat{u}, \hat{\rho}) \in H^{1}(\mathbb{R}) \times L^{2}(\mathbb{R})$, there exists some positive constant $C$ depends only on $E_{1}$, such that,

$$
d((u, \rho),(\hat{u}, \hat{\rho})) \leq C\left(\|u-\hat{u}\|_{H^{1}}+\left\|(u-\hat{u}) e^{-|x|}\right\|_{L^{1}}+\|\rho-\hat{\rho}\|_{L^{2}}+\left\|(\rho-\hat{\rho}) e^{-|x|}\right\|_{L^{1}}\right) .
$$

For any $u, \hat{u} \in H^{1}(\mathbb{R})$ and $\rho, \hat{\rho} \in L^{2}(\mathbb{R})$, there exists some constant $C>0$ depends only on $E_{1}$, such that,

$$
\begin{gathered}
\left\|(u-\hat{u}) e^{-|x|}\right\|_{L^{1}} \leq C \cdot d((u, \rho),(\hat{u}, \hat{\rho})), \\
\mid \text { meas } \lambda-\text { meas } \hat{\lambda} \mid \leq C \cdot d((u, \rho),(\hat{u}, \hat{\rho})),
\end{gathered}
$$




$$
\sup _{\|f\|_{\mathcal{C}^{1} \leq 1}}\left|\int f d \mu-\int f d \hat{\mu}\right| \leq C \cdot d((u, \rho),(\hat{u}, \hat{\rho})),
$$

where $\lambda, \hat{\lambda}$ are the measures with densities $\rho e^{-|x|}$ and $\hat{\rho} e^{-|x|}$ with respect to Lebesgue measure, and $\mu, \hat{\mu}$ are the measures with densities $\left(\left(u_{x}\right)^{2}+\rho^{2}\right) e^{-|x|}$ and $\left(\left(\hat{u}_{x}\right)^{2}+\hat{\rho}^{2}\right) e^{-|x|}$ with respect to Lebesgue measure.

Acknowledgements. The work of Cai is partially supported by the National Natural Science Foundation of China-NSAF (No. 11271305, 11531010) and the China Scholarship Council No. 201506310110 as an exchange graduate student at Georgia Institute of Technology.

\section{REFERENCES}

[1] A. Bressan and G. Chen, Generic regularity of conservative solutions to a nonlinear wave equation, Ann. I. H. Poincaré-AN (2016), http://dx.doi.org/10.1016/j.anihpc.2015.12.004.

[2] A. Bressan and G. Chen, Lipschitz metrics for a class of nonlinear wave equations, submitted, available at arXiv:1506.06310 [math.AP].

[3] H. Cai, G. Chen, R. M. Chen and Y. Shen, Lipschitz metric for the Novikov equation, preprint.

[4] H. Cai, G. Chen, Y. Shen and Z. Tan, Generic Regularity and Lipschitz Metric for the Hunter-Saxton type equations, submitted, available at http://www.csun.edu/ yshen/HS4.pdf.

[5] A. Constantin and R. Ivanov, On an integrable two-component Camassa-Holm shallow water system, Phys. Lett. A 372 (2008) 7129-7132.

[6] K. Grunert, Blow-up for the two-component Camassa-Holm system, available at arXiv:1401.6569 [math.AP].

[7] K. Grunert, H. Holden and X. Raynaud, Global solutions for the two-component Camassa-Holm system, Comm. Partial Differential Equations 37 (2012) 2245-2271.

[8] K. Grunert, H. Holden and X. Raynaud, A continuous interpolation between conservative and dissipative solutions for the two-component Camassa-Holm system, Forum Math. 3 (2015) 73 pages.

[9] K. Grunert, H. Holden and X. Raynaud, Lipschitz metric for the two-component Camassa-Holm system, available at arXiv:1306.6822 [math.AP].

[10] C. Guan and Z. Yin, Global existence and blow-up phenomena for an integrable two-component CamassaHolm shallow water system, J. Differential Equations 248 (2010) 2003-2014.

[11] G. Gui and Y. Liu, On the global existence and wave breaking criteria for the two-component Camassa-Holm system, J. Func. Anal. 258 (2010) 4251-4278.

[12] M. J. Li and Q. T. Zhang, Generic regularity of conservative solutions to Camassa-Holm type equations. 2015. < hal-01202927>.

[13] M. J. Li and Q. Zhang, Uniqueness of conservative solutions to the two-component Camassa-Holm system via characteristics, 2015. < hal-01183748>.

Hong CAi

School of Mathematical Sciences, Xiamen University, Fujian, Xiamen, 361005, China, and School of Mathematics, Georgia Institute of Technology, Atlanta, Ga 30332, USA.

E-mail address: caihong19890418@163.com

Geng Chen

Department of Mathematics, University of Kansas, Lawrence, KS 66045

E-mail address: gengchen@ku.edu

YANNAN SHEN

Department of Mathematics, California Sate University, Northridge, CA 91330

E-mail address: yannan.shen@csun.edu 